\title{
Unconventional Topological Insulators from Extended Topological Band Degeneracies
}

\author{
Zhongbo $\operatorname{Yan}^{1, *}$ \\ ${ }^{1}$ School of Physics, Sun Yat-Sen University, Guangzhou 510275, China
}

\begin{abstract}
A general and beautiful picture for the realization of topological insulators is that the mass term of the Dirac model has a nodal surface wrapping one Dirac point. We show that this geometric picture based on Dirac points can be generalized to extended band degeneracies with nontrivial topological charges. As the nontrivial topological charges force the extended band degeneracies to be created or annihilated in pairs, when the nodal surface of mass wraps one such extended band degeneracy, the resulting gapped phase must be topologically nontrivial since it cannot adiabatically be deformed into a topologically trivial atomic insulator without closing the energy gap. We use nodal lines carrying a nontrivial $Z_{2}$ monopole charge in three dimensions to illustrate the physics. Notably, because the wrapping surfaces for an extended band degeneracy are diverse, we find that this generalization can bring topological insulators with unconventional pattern of boundary states.
\end{abstract}

Dirac models paly a fundamental role in the description of topological insulators and superconductors[1-5]. In $D$ dimensions, their tight-binding forms are simply given by

$$
H(\mathbf{k})=\sum_{i=1}^{D} \lambda_{i} \sin k_{i} \Gamma_{i}+m(\mathbf{k}) \Gamma_{D+1},
$$

with the matrices $\Gamma_{1,2, \ldots, D+1}$ satisfying the Clifford algebra, i.e., $\left\{\Gamma_{i}, \Gamma_{j}\right\}=2 \delta_{i j} . m(\mathbf{k})$ is an even function of the momentum and known as the Dirac mass. When $m(\mathbf{k})$ vanishes identically, the energy bands touch and form Dirac points at timereversal invariant momenta. A universal rule for nonaccidental Dirac points is that they must be created or annihilated in pairs, known as the fermion-doubling theorem[6]. When the existence of some symmetries forces the absence of additional mass terms which anti-commute with all terms in Eq.(1), i.e., the Dirac mass term $m(\mathbf{k}) \Gamma_{D+1}$ is unique due to symmetry constraint, a strong topological insulator or superconductor is realized when the zeros of $m(\mathbf{k})$, dubbed mass nodal surface (MNS) in this paper, wraps only one of the Dirac points. The underlying mechanism is simply because the resultant gapped phase for such a configuration cannot continuously be deformed into the topologically trivial atomic limit $\left(\lambda_{i}=0\right.$ and $m(\mathbf{k})=m$ with $m$ a nonzero constant) without closing the energy gap.

Band degeneracies, however, are not restricted to the form of point. They can also be extended in the momentum space and form very rich structures. For instance, one-dimensional band degeneracy, also known as nodal line, can display structures of the form from rings[7-10] and chains[11-14] to exotic links[15-19] and knots[20]. Over the past few years, materials with such one-dimensional band degeneracies near the Fermi energy have attracted great interest[21-23]. Similarly to the Dirac point, an extended band degeneracy requires appropriate crystal symmetries to protect its stability. By breaking the protecting symmetry, it may be split into some lower-dimensional band degeneracies or completely gapped out. With the Dirac models in mind, the latter situation attracts us to investigate the underlying topological property of the resultant gapped phase for which the mass term has a nodal surface wrapping only one extended band degeneracy.

*yanzhb5@mail.sysu.edu.cn
Extended band degeneracies, unlike Dirac points, are in principle unnecessary to obey the fermion-doubling theorem. As a result, we need to divide them into two classes. For the first class, they do not carry a global topological charge and can be singly created or annihilated by tuning the Hamiltonian parameters without breaking the protecting symmetries[10]. For the second class, they carry a nontrivial global topological charge and therefore must be created or annihilated in pairs like the Dirac points[10, 24-26]. For the first class, it is obvious that the resultant gapped phase must be topologically trivial because we can first deform the extended band degeneracy to vanish and then deform the wrapping MNS to vanish without closing the bulk gap. In other words, the insulator is adiabatically connected to a topologically trivial atomic insulator. For the second class, it is obvious that the adiabatic path to the atomic insulator is obstructed, just like the scenario in conventional topological insulators, therefore, the resultant gapped phase must be topologically nontrivial. Because of the distinction in dimensions for the wrapped band degeneracy, it is natural to expect that this new scenario may bring new types of topological phases with unconventional pattern of boundary states. In this work, we use one-dimensional nodal lines carrying a nontrivial $Z_{2}$ monopole charge in three dimensions as a concrete example to demonstrate the above general arguments.

Nodal lines protected by a $Z_{2}$ monopole charge- Similarly to the Dirac model in Eq.(1), we divide the Hamiltonian into two parts, i.e., $H=H_{\mathrm{NL}}+H_{\mathrm{M}}$, with $H_{\mathrm{NL}}$ describing the part realizing topological band degeneracies and $H_{\mathrm{M}}$ the mass term gapping out the band degeneracies. $H_{\mathrm{NL}}$ and $H_{\mathrm{M}}$ satisfy $\left\{H_{\mathrm{NL}}, H_{\mathrm{M}}\right\}=0$. In Ref.[10], Fang et al showed that when spin-orbit coupling is negligible and a combination of inversion symmetry $(\mathrm{P})$ and time-reversal symmetry $(\mathrm{T})$ is present, nodal lines carrying a nontrivial $Z_{2}$ monopole charge can be realized in a four-band minimal model. Here we consider a tight-binding generalization of the model in Ref.[10], which reads

$$
\begin{aligned}
H_{\mathrm{NL}}(\mathbf{k})= & \lambda_{z} \sin k_{z} s_{z}+\lambda_{x} \sin k_{x} s_{x}+\lambda_{y} \sin k_{y} \sigma_{y} s_{y} \\
& +\left(t_{0}-t \cos k_{x}-t \cos k_{y}-t \cos k_{z}\right) \sigma_{x} s_{x},
\end{aligned}
$$

where $\sigma_{i}$ and $s_{i}$ act on certain pseudo-spin degrees of freedom. For notational simplicity, the lattice constant is set to unit throughout this work. This Hamiltonian has both $P$ and 
$T$, with $P=\sigma_{z} s_{y}$ and $T=\sigma_{z} s_{y} K$ ( $K$ denotes the complex conjugation). Since $[P, T]=0$ and $(P T)^{2}=1$, the energy bands of the Hamiltonian do not have the double degeneracy as in a spinful system with both $P$ and $T$. However, here $T^{2}$ also gives -1 , so Kramers degeneracy are still present at timereversal invariant momenta.

The energy spectra for $H_{\mathrm{NL}}(\mathbf{k})$ read

$$
E(\mathbf{k})= \pm \sqrt{\left(\lambda_{z} \sin k_{z}\right)^{2}+(A(\mathbf{k}) \pm B(\mathbf{k}))^{2}}
$$

where $A(\mathbf{k})=\sqrt{\left(\lambda_{x} \sin k_{x}\right)^{2}+\left(\lambda_{y} \sin k_{y}\right)^{2}}$ and $B(\mathbf{k})=\left(t_{0}-\right.$ $t \cos k_{x}-t \cos k_{y}-t \cos k_{z}$ ). The nodal lines are located at either the $k_{z}=0$ plane or the $k_{z}=\pi$ plane. At the $k_{x}=0$ plane, they are determined by $\sqrt{\left(\lambda_{x} \sin k_{x}\right)^{2}+\left(\lambda_{y} \sin k_{y}\right)^{2}}= \pm\left(t_{0}-t-\right.$ $\left.t \cos k_{x}-t \cos k_{y}\right)$. At the $k_{x}=\pi$ plane, they are determined by $\sqrt{\left(\lambda_{x} \sin k_{x}\right)^{2}+\left(\lambda_{y} \sin k_{y}\right)^{2}}= \pm\left(t_{0}+t-t \cos k_{x}-t \cos k_{y}\right)$. Without losing generality, in this work we consider the simplest situation with only two concentric nodal lines located at the $k_{z}=0$ plane, as shown in Fig.1. From the evolution of nodal lines presented in Figs.1(a)-(e), one can infer the fact that for this Hamiltonian, a nodal line enclosing a timereversal invariant momentum cannot shrink to a point and vanish. In Ref.[10], Fang et al explained that the underlying reason is because the nodal line carries a nontrivial $Z_{2}$ monopole charge. Without explicitly calculating this topological invariant in terms of the wave functions of occupied states, it in fact can be intuitively understood by noting the fact that when the nodal line shrinks to a point at a time-reversal invariant momentum, it reduces to a Dirac point at this critical situation (see Eq.(2)), suggesting that the $Z_{2}$ monopole charge of the nodal line is inherited from the Dirac point. It is noteworthy that while nodal lines carrying nontrivial and trivial $Z_{2}$ monopole charges display remarkable distinction in stability, the associated surface states, however, take the same characteristic drumhead form, as shown in Fig.1(f).

MNS of the sphere structure - Let us first investigate the situation that the wrapping MNS is of the sphere structure. To be specific, we let

$$
H_{\mathrm{M}}(\mathbf{k})=\left(m_{0}-\cos k_{x}-\cos k_{y}-\cos k_{z}\right) \sigma_{x} s_{y} .
$$

It is readily checked that the Pauli matrix form of $H_{\mathrm{M}}$ is the only possibility that anti-commutes with all terms in $H_{\mathrm{NL}}$. One can also check that $\left[T, H_{\mathrm{M}}\right]=0$ but $\left[P, H_{\mathrm{M}}\right] \neq 0$, suggesting that the introduction of $H_{\mathrm{M}}$ does not break the time-reversal symmetry, but breaks the inversion symmetry and their combinational symmetry (the $P T$ symmetry which forces the Hamiltonian to be real), consequently gapping out the nodal lines.

Let us consider that the MNS determined by $\sum_{i=x, y, z} \cos k_{i}=$ $m_{0}$ wraps the nodal line with smaller size. For concreteness, we let $m_{0}=t_{0}=2.5$, which naturally satisfies the requirement, as shown in Fig.2(a). Apparently, because the two nodal lines can only be annihilated in pairs, no matter how we deform the MNS, inwardly or outwardly, it will inevitably cross one of the nodal lines before it gets vanished. In other words, the MNS cannot vanish without closing the energy gap. This simple fact indicates that the adiabatic path to a topologically trivial atomic insulator is obstructed, so the configura-

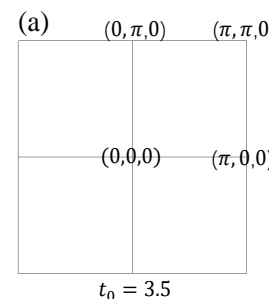

(d)

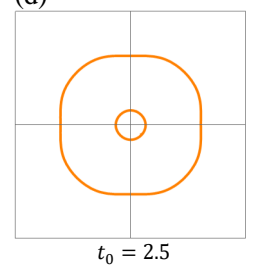

(b)

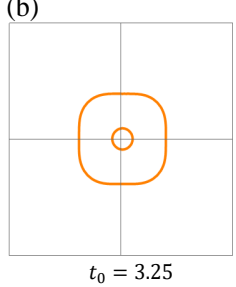

(e)

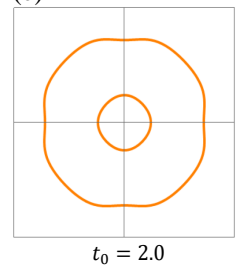

(c)
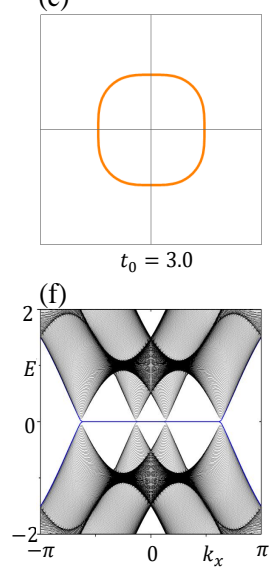

FIG. 1. (a)-(e) The evolution of $Z_{2}$-monopole-charge nodal lines in the $k_{z}=0$ plane. (a) no nodal lines exist, (b) two concentric nodal lines emerge, (c) one of the nodal lines shrinks to a point, (d) the point re-expands to a line, (e) the size of nodal lines increases further. (f) Energy spectra for a geometry with open boundary condition in the $z$ direction and periodic boundary condition in both $x$ and $y$ directions. $k_{y}=0, t_{0}=2.5$, and the system size in the $z$ direction is $L_{z}=100$. Characteristic drumhead surface states appear within the projections of the nodal lines. Common parameters: $\lambda_{x, y, z}=t=1$.

tion shown in Fig.2(a) must correspond to a topological insulator. By investigating the boundary states, we find that each open surface indeed harbors a single Dirac cone, as shown in Figs.2(b)(c). Because of $T^{2}=-1$ as mentioned previously, the Dirac cones are centered at the time-reversal invariant momentum $\bar{\Gamma}$ of the surface Brillouin zone. Just like in a spinful topological insulator, here the Dirac cones are also robust against perturbations respecting time-reversal symmetry. These results demonstrate the realization of a topological insulator with robust boundary states. For comparison, we have also investigated the configuration with the MNS wrapping both nodal lines (see the inset of Fig.2(d)). Owing to the $Z_{2}$ nature of the nodal lines, we find that as expected there is no boundary states connecting the conduction and valence bands, demonstrating that the configuration corresponds to a topologically trivial insulator. Nevertheless, we find that Dirac cones still appear on the $z$-normal surfaces, though their energy are shifted away from the gap, as shown in Fig.2(d).

MNS of the torus structure - For a nodal line, the wrapping MNS can also take the torus structure. For simplicity, we first consider a mass term of the form

$$
H_{\mathrm{M}}(\mathbf{k})=\left(m_{0}-\cos k_{x}-\cos k_{y}\right) \sigma_{x} s_{y} .
$$

Because of the periodicity of the Brillouin zone, $H_{\mathrm{M}}$ gives a torus-form MNS for $-2<m<2$. Let us again consider that the MNS encloses the nodal line with smaller size, as shown in Fig.3(a). It is easy to see that this configuration is adiabatically connected to the configuration in Fig.2(a), so it must also realize a topological insulator. Notably, we find that the boundary states on the $z$-normal surfaces for this configuration no longer take the Dirac cone structure, instead they take a bowl structure, as shown in Fig.3(b). Anyway, the boundary 
(a)
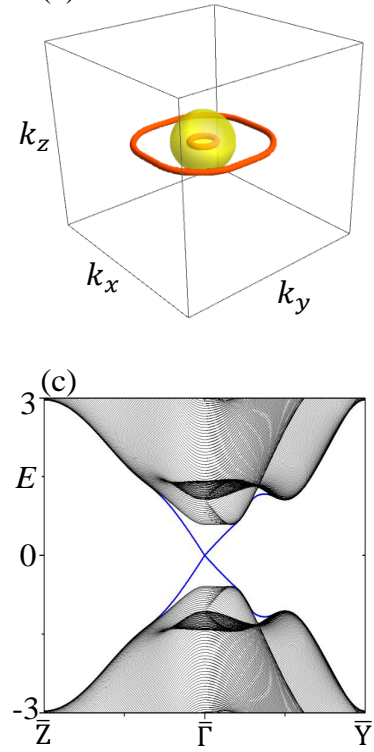
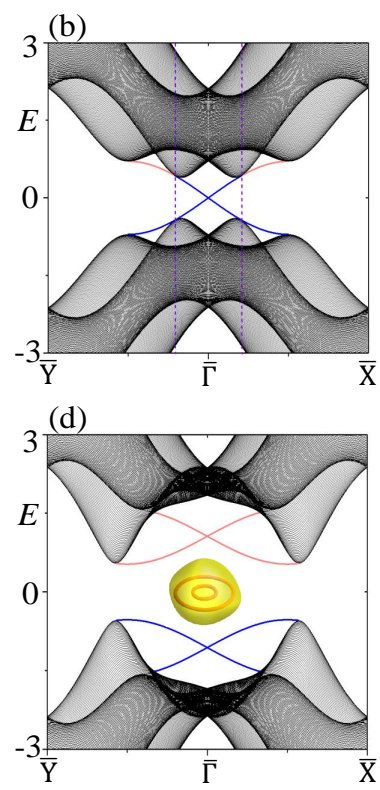

(a)
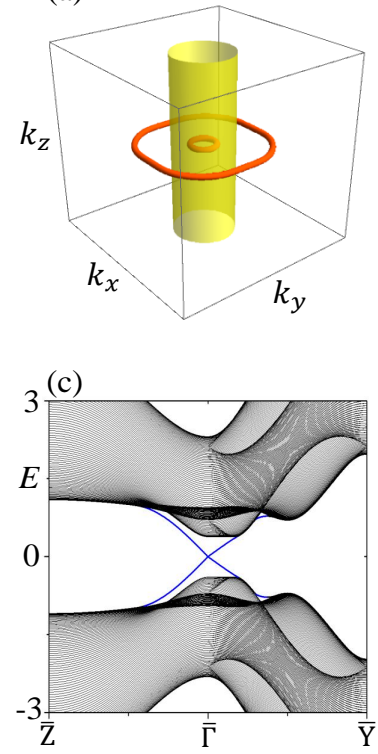
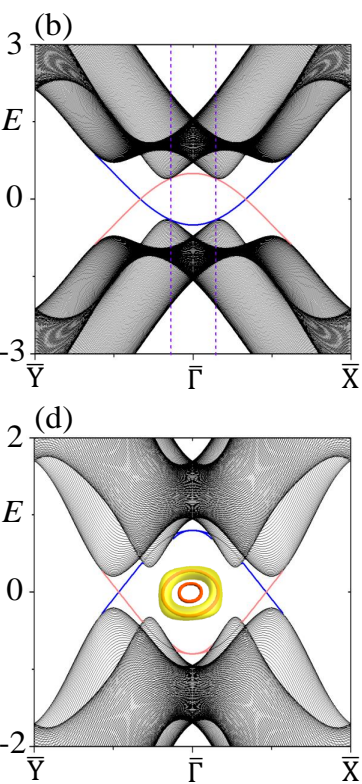

FIG. 2. (a) A sphere-form MNS (yellow) wraps the nodal line (red) with smaller size. (b) Between the two dashed purple lines, the in-gap dispersions are doubly degenerate, corresponding to the existence of a single Dirac cone on each $z$-normal surface. Outside, the blue and red lines are without degeneracy and represent boundary states on the lower and upper surface, respectively. (c) Each $x$ normal or $y$-normal surface also harbors a single Dirac cone, here we have only presented the results for $x$-normal surfaces because the system has rotational symmetry in the $x y$ plane. (d) When the MNS wraps both nodal lines (the inset), the configuration realizes a trivial insulator but with surface Dirac cones coexisting with bulk states on the $z$-normal surfaces. The parameters are: $\lambda_{x, y, z}=t=1$ and $t_{0}=m_{0}=2.5$ in (a)(b)(c); $\lambda_{z}=t=1, \lambda_{x}=\lambda_{y}=0.5, t_{0}=2.5$, and $m_{0}=1.5$ in (d); $L_{z}=100$ in (b)(d), and $L_{x}=100$ in (c).

states still connect the conduction and valence bands, confirming the nontrivial band topology. To the best of our knowledge, such bowl-like surface states have previously only been found in magnetic Hopf insulators[16, 27, 28]. This coincidence has a natural explanation. In the Hopf insulators, the bowl-like surface states are tied to the underlying link structure. In Fig.3(a), the nodal line with larger size and the torusform MNS in fact form an extra link structure similar to that in the Hopf insulator. In spite of similarity, we need to emphasize that there also exist remarkable difference between them. In the Hopf insulators, a bowl-like surface state is of one-band nature and spin-polarized due to the absence of time-reversal symmetry[16, 27, 28]. In Fig.3(b), while the bowl-like surface state on each surface also looks like of one-band nature, the time-reversal symmetry forces the pseudo-spin and the momentum to be locked. That is, the bowl-like surface states in Fig.3(b) exhibit nontrivial pseudo-spin textures just like the Dirac surface states. An intuitive understanding of the bowl structure and the nontrivial pseudo-spin textures is that each bowl-like surface state in the gap corresponds to one part of a Dirac cone, with the other part containing the Dirac point buried in the bulk states.
FIG. 3. (a) A torus-form MNS (yellow) wraps the nodal line (red) with smaller size. (b) Between the two dashed purple lines, the dispersions in red color and blue color are doubly degenerate, which correspond to trivial boundary states on the upper and lower $z$-normal surface, respectively. Outside, the in-gap dispersions are without degeneracy and correspond to topological boundary states. (c) Each $x$-normal surface (also for $y$-normal surfaces) harbors a single Dirac cone. (d) Boundary states on the $z$-normal surfaces for the configuration that the torus-form MNS encloses the nodal line with larger size (the inset). The parameters are: $\lambda_{x, y, z}=t=1, t_{0}=2.5$ and $m_{0}=1.5$ in (a)(b)(c); $\lambda_{z}=t=1, \lambda_{x}=\lambda_{y}=0.5, t_{0}=2.5, B_{0}=2.0$ and $\delta=0.2$ in (d); $L_{z}=100$ in (b)(d), and $L_{x}=100$ in (c).

For $x$-normal and $y$-normal surfaces, we find that each surface still harbors a single Dirac cone, as shown in Fig.3(c). The coexistence of surface Dirac cones and bowl-like topological surface states reveals a new type of topological insulating phases unexplored before. Notably, the total Hamiltonian $H=H_{\mathrm{NL}}+H_{\mathrm{M}}$ still only involves at most nearest-neighbor hoppings, its simplicity holds great promise for the realization of this type of topological insulators, both in real materials and in artificial systems.

While from the above analysis it is apparent that a topological insulator should also be realized when the MNS encloses only the nodal line with larger size, we demonstrate this explicitly for completeness. To be specific, we consider a mass term of the form

$$
H_{\mathrm{M}}(\mathbf{k})=\left[\sin ^{2} k_{z}+\left(B_{0}-\sum_{i=x, y, z} \cos k_{i}\right)^{2}-\delta\right] \sigma_{x} s_{y},
$$

where $B_{0}$ and $\delta$ are two real parameters controlling the position and the size of the MNS. Fig.3(d) shows that the boundary states on the $z$-normal surfaces also take the bowl structure when the MNS forms a torus enclosing the nodal line with larger size (see the inset). The result can be simply understood by noting the torus in Fig.3(a) can be continuously deformed to the torus in Fig.3(d). In addition, one can find 
(a)

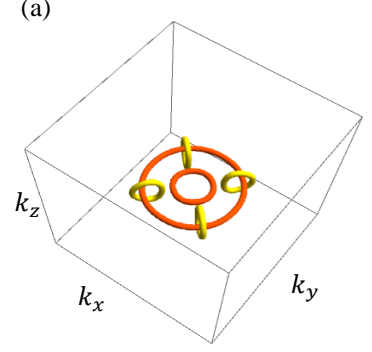

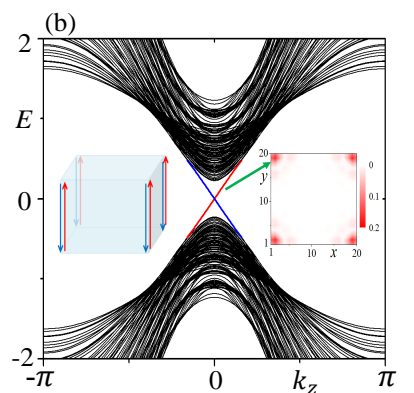

FIG. 4. (a) Nodal lines from $H_{\mathrm{NL}}$ and $H_{\mathrm{M}}$ form link structure. (b) The in-gap dispersions in red color (up-moving states) and blue color (down-moving states) are of four-fold degeneracy, corresponding to four pairs of gapless helical states localized on the four hinges. The left inset is a schematic diagram for the distribution of the gapless helical states, and the right inset shows the probability density profiles of the four branches of upmoving states at $k_{z}=0.1$. The parameters are: $\lambda_{z}=t=1, \lambda_{x}=\lambda_{y}=0.5, t_{0}=2.5, B_{0}=2.0, \delta=0.3$, and $L_{x}=L_{y}=20$.

from Fig.3(d) that the cross section of the MNS for a given toroidal angle links with the nodal line.

Generalization - It is apparent that the geometry picture exemplified in three dimensions is also applied to dimensions higher than three. While the spatial dimension of real materials is bounded by three, topological insulators in dimensions $D \geq 4$ can be simulated in terms of synthetic dimensions which are realized by certain controllable internal degrees of freedom or periodic parameters[29-32]. Notably, extended band degeneracy become more common and diverse in higher dimensions, which consequently allows the formation of more exotic configurations for the MNS and extended band degeneracy.

Another interesting direction for generalization is the dimension of the MNS. This direction is relevant to systems with more than one symmetry-allowed mass term. As mentioned previously, such a situation implies a trivial insulator. However, the triviality is to the first-order level. Very recently, the concept dubbed higher-order topological insulator indicates that many classes of insulators which were believed to be trivial can be topologically nontrivial in a higherorder sense[33-39]. That is, topological gapless states can appear at some lower-dimensional boundaries even when the one-dimensional lower boundaries do not harbor topological gapless states.

It is readily seen that when $H_{M}$ contains at least two anticommuting terms and $\left\{H_{\mathrm{NL}}, H_{\mathrm{M}}\right\}=0$ is still hold, the MNS becomes the band degeneracy of $H_{\mathrm{M}}$ and its dimension will be reduced. The dimension reduction of the MNS, however, does not rule out the possibility for the MNS and extended band degeneracy to form configurations that are not adiabatically connected to the topologically trivial atomic limit. For instance, when two mass terms are allowed by symmetry in three dimensions, the MNSs also become lines which can form topological link structures with the nodal lines from $H_{\mathrm{NL}}$. As an example, we consider the following Hamiltonian,

$$
\begin{aligned}
H(\mathbf{k})= & H_{\mathrm{NL}} \otimes \tau_{z}+\left[\sin ^{2} k_{z}+\left(B_{0}-\sum_{i=x, y, z} \cos k_{i}\right)^{2}-\delta\right] \sigma_{x} s_{y} \tau_{z} \\
& +\left(\cos k_{x}-\cos k_{y}\right) \tau_{x},
\end{aligned}
$$

where $\tau_{i}$ act on another pseudospin. While each nodal line is doubled due to the introduction of a new pseudospin, they cannot be annihilated with their degenerate partners if the pseudospin is conserved. Because of the doubling, it is readily checked that the last two mass terms do not break the timereversal symmetry and chiral symmetry of $H_{\mathrm{NL}} \otimes \tau_{z}$ (the chiral operator is $\tau_{y}$ ). As shown in Fig.4(a), for appropriate $B_{0}$ and $\delta$, the nodal lines originated from the mass terms form link structure with the nodal lines from $H_{\mathrm{NL}} \otimes \tau_{z}$. By considering a geometry with open boundary condition in the $x y$ plane and periodic boundary condition in the $z$ direction, we find that gapless helical states appear at the one-dimensional hinges of the geometry (see Fig.4(b)), indicating that the link configuration in Fig.4(a) realizes a higher-order topological insulator.

Discussions and Conclusions. - It is easy to see that the decomposition $H_{\mathrm{NL}}+H_{\mathrm{M}}$ can also be decomposed as $H_{\mathrm{D}}+H_{\mathrm{SB}}$, where $H_{\mathrm{D}}$ takes the Dirac form given in Eq.(1) and $H_{\mathrm{SB}}$ denotes symmetry-breaking terms. Therefore, the geometric picture developed in this work can also be applied to analyze the topological property of insulators without certain symmetries, e.g., insulators without inversion symmetry. As is known, when the inversion symmetry is broken, the topological property of a time-reversal invariant insulator can no longer be simply determined by the parity at time-reversal invariant momenta[40]. The geometric picture developed in this work, however, is hold as long as that decompositions like $H_{\mathrm{NL}}+H_{\mathrm{M}}$ with $\left\{H_{\mathrm{NL}}, H_{\mathrm{M}}\right\}=0$ is possible.

In summary, we have developed a new geometric picture for topological insulators on the basis of extended band degeneracies. Guided by it, we have revealed the existence of new types of topological insulators with unconventional pattern of boundary states through concrete examples in three dimensions. The simplicity of the developed geometric picture allows it to be taken as a guiding principle for the discovery and design of new types of topological insulators.

This work is supported by the Startup Grant (No. 7413018841219) and the National Science Foundation of China (Grant No.11904417).
[1] M. Z. Hasan and C. L. Kane, "Colloquium : Topological insulators,” Rev. Mod. Phys. 82, 3045-3067 (2010).

[2] Xiao-Liang Qi and Shou-Cheng Zhang, "Topological insulators and superconductors,” Rev. Mod. Phys. 83, 1057-1110 (2011).
[3] Shinsei Ryu, Andreas Schnyder, Akira Furusaki, and Andreas Ludwig, "Topological insulators and superconductors: ten-fold way and dimensional hierarchy," New J. Phys. 12, 065010 (2010). 
[4] Shun-Qing Shen, Topological Insulators: Dirac Equation in Condensed Matters, Vol. 174 (Springer Science \& Business Media, 2013).

[5] B Andrei Bernevig and Taylor L Hughes, Topological insulators and topological superconductors (Princeton University Press, 2013).

[6] H.B. Nielsen and M. Ninomiya, "Absence of neutrinos on a lattice: (i). proof by homotopy theory,” Nuclear Physics B $\mathbf{1 8 5}$, $20-40$ (1981).

[7] A. A. Burkov, M. D. Hook, and Leon Balents, "Topological nodal semimetals," Phys. Rev. B 84, 235126 (2011).

[8] Rui Yu, Hongming Weng, Zhong Fang, Xi Dai, and Xiao Hu, "Topological node-line semimetal and dirac semimetal state in antiperovskite $\mathrm{cu}_{3}$ PdN," Phys. Rev. Lett. 115, 036807 (2015).

[9] Youngkuk Kim, Benjamin J. Wieder, C. L. Kane, and Andrew M. Rappe, "Dirac line nodes in inversion-symmetric crystals," Phys. Rev. Lett. 115, 036806 (2015).

[10] Chen Fang, Yige Chen, Hae-Young Kee, and Liang Fu, "Topological nodal line semimetals with and without spin-orbital coupling," Phys. Rev. B 92, 081201 (2015).

[11] Tomáš Bzdušek, QuanSheng Wu, Andreas Rüegg, Manfred Sigrist, and Alexey A. Soluyanov, "Nodal-chain metals," Nature 538, 75-78 (2016).

[12] Rui Yu, Quansheng Wu, Zhong Fang, and Hongming Weng, "From nodal chain semimetal to weyl semimetal in hfc," Phys. Rev. Lett. 119, 036401 (2017).

[13] Qinghui Yan, Rongjuan Liu, Zhongbo Yan, Boyuan Liu, Hongsheng Chen, Zhong Wang, and Ling Lu, "Experimental discovery of nodal chains," Nature Physics 14, 461-464 (2018).

[14] Cheng Gong, Yuee Xie, Yuanping Chen, Heung-Sik Kim, and David Vanderbilt, "Symmorphic intersecting nodal rings in semiconducting layers," Phys. Rev. Lett. 120, 106403 (2018).

[15] Wei Chen, Hai-Zhou Lu, and Jing-Min Hou, "Topological semimetals with a double-helix nodal link," Phys. Rev. B 96, 041102 (2017).

[16] Zhongbo Yan, Ren Bi, Huitao Shen, Ling Lu, Shou-Cheng Zhang, and Zhong Wang, "Nodal-link semimetals," Phys. Rev. B 96, 041103 (2017).

[17] Motohiko Ezawa, "Topological semimetals carrying arbitrary hopf numbers: Fermi surface topologies of a hopf link, solomon's knot, trefoil knot, and other linked nodal varieties," Phys. Rev. B 96, 041202 (2017).

[18] Guoqing Chang, Su-Yang Xu, Xiaoting Zhou, Shin-Ming Huang, Bahadur Singh, Baokai Wang, Ilya Belopolski, Jiaxin Yin, Songtian Zhang, Arun Bansil, Hsin Lin, and M. Zahid Hasan, "Topological hopf and chain link semimetal states and their application to $\mathrm{co}_{2} \mathrm{MnGa}$," Phys. Rev. Lett. 119, 156401 (2017).

[19] Erchan Yang, Biao Yang, Oubo You, Hsun-chi Chan, Peng Mao, Qinghua Guo, Shaojie Ma, Lingbo Xia, Dianyuan Fan, Yuanjiang Xiang, and Shuang Zhang, "Observation of non-Abelian nodal links in photonics," arXiv e-prints , arXiv:1910.01345 (2019), arXiv:1910.01345 [physics.optics].

[20] Ren Bi, Zhongbo Yan, Ling Lu, and Zhong Wang, "Nodal-knot semimetals," Phys. Rev. B 96, 201305 (2017).

[21] Chen Fang, Hongming Weng, Xi Dai, and Zhong Fang, "Topological nodal line semimetals," Chinese Physics B 25, 117106 (2016).

[22] Shuo-Ying Yang, Hao Yang, Elena Derunova, Stuart S. P. Parkin, Binghai Yan, and Mazhar N. Ali, "Symmetry demanded topological nodal-line materials," Advances in Physics: X 3, 1414631 (2018).
[23] Sebastian Klemenz, Shiming Lei, and Leslie M. Schoop, "Topological semimetals in square-net materials," Annual Review of Materials Research 49, 185-206 (2019).

[24] Tomá š Bzdušek and Manfred Sigrist, "Robust doubly charged nodal lines and nodal surfaces in centrosymmetric systems," Phys. Rev. B 96, 155105 (2017).

[25] Junyeong Ahn, Dongwook Kim, Youngkuk Kim, and BohmJung Yang, "Band topology and linking structure of nodal line semimetals with $Z_{2}$ monopole charges," Phys. Rev. Lett. 121, 106403 (2018).

[26] Zhida Song, Tiantian Zhang, and Chen Fang, "Diagnosis for nonmagnetic topological semimetals in the absence of spinorbital coupling," Phys. Rev. X 8, 031069 (2018).

[27] Joel E. Moore, Ying Ran, and Xiao-Gang Wen, "Topological surface states in three-dimensional magnetic insulators," Phys. Rev. Lett. 101, 186805 (2008).

[28] A. Alexandradinata, Aleksandra Nelson, and Alexey A. Soluyanov, "Teleportation of Berry curvature on the surface of a Hopf insulator," arXiv e-prints , arXiv:1910.10717 (2019), arXiv:1910.10717 [cond-mat.str-el].

[29] A. Celi, P. Massignan, J. Ruseckas, N. Goldman, I. B. Spielman, G. Juzeliūnas, and M. Lewenstein, "Synthetic gauge fields in synthetic dimensions," Phys. Rev. Lett. 112, 043001 (2014).

[30] H. M. Price, O. Zilberberg, T. Ozawa, I. Carusotto, and N. Goldman, "Four-dimensional quantum hall effect with ultracold atoms," Phys. Rev. Lett. 115, 195303 (2015).

[31] Tomoki Ozawa, Hannah M. Price, Nathan Goldman, Oded Zilberberg, and Iacopo Carusotto, "Synthetic dimensions in integrated photonics: From optical isolation to four-dimensional quantum hall physics,” Phys. Rev. A 93, 043827 (2016).

[32] Tomoki Ozawa and Hannah M. Price, "Topological quantum matter in synthetic dimensions," Nature Reviews Physics 1, 349-357 (2019).

[33] Wladimir A Benalcazar, B Andrei Bernevig, and Taylor L Hughes, "Quantized electric multipole insulators," Science 357, 61-66 (2017).

[34] Frank Schindler, Ashley M. Cook, Maia G. Vergniory, Zhijun Wang, Stuart S. P. Parkin, B. Andrei Bernevig, and Titus Neupert, "Higher-order topological insulators," Science Advances 4 (2018), 10.1126/sciadv.aat0346.

[35] M. Sitte, A. Rosch, E. Altman, and L. Fritz, "Topological insulators in magnetic fields: Quantum hall effect and edge channels with a nonquantized $\theta$ term,' Phys. Rev. Lett. 108, 126807 (2012).

[36] Fan Zhang, C. L. Kane, and E. J. Mele, "Surface state magnetization and chiral edge states on topological insulators," Phys. Rev. Lett. 110, 046404 (2013).

[37] Wladimir A. Benalcazar, B. Andrei Bernevig, and Taylor L. Hughes, "Electric multipole moments, topological multipole moment pumping, and chiral hinge states in crystalline insulators," Phys. Rev. B 96, 245115 (2017).

[38] Zhida Song, Zhong Fang, and Chen Fang, " $(d-2)$-dimensional edge states of rotation symmetry protected topological states," Phys. Rev. Lett. 119, 246402 (2017).

[39] J. Langbehn, Yang Peng, L. Trifunovic, Felix von Oppen, and Piet W. Brouwer, "Reflection-symmetric second-order topological insulators and superconductors," Phys. Rev. Lett. 119, 246401 (2017).

[40] Liang Fu and C. L. Kane, "Topological insulators with inversion symmetry,” Phys. Rev. B 76, 045302 (2007). 